\title{
Nuclear Magnetic Resonance Study of the Rotational Motion and the Phase Transition in $\mathrm{LiBH}_{4}$
}

\author{
Alexander V. Skripov, ${ }^{*} \dagger$ Alexei V. Soloninin, ${ }^{\dagger}$ Yaroslav Filinchuk, ${ }^{\ddagger}$ and Dmitry Chernyshov \\ Institute of Metal Physics, Ural Division of the Russian Academy of Sciences, S. Kovalevskoi 18, \\ Ekaterinburg 620041, Russia, and Swiss-Norwegian Beam Lines at ESRF, BP-220, 38043, Grenoble, France
}

Received: July 29, 2008; Revised Manuscript Received: September 25, 2008

\begin{abstract}
To study the rotational motion of $\mathrm{BH}_{4}$ tetrahedra in $\mathrm{LiBH}_{4}$, we have measured the ${ }^{1} \mathrm{H}$ and ${ }^{11} \mathrm{~B}$ nuclear magnetic resonance spectra and spin-lattice relaxation rates in this compound over wide ranges of temperature (92-424 $\mathrm{K})$ and resonance frequency $\left(14-90 \mathrm{MHz}\right.$ for ${ }^{1} \mathrm{H}$ and $14-28 \mathrm{MHz}$ for $\left.{ }^{11} \mathrm{~B}\right)$. In the low-temperature (orthorhombic) phase of $\mathrm{LiBH}_{4}$, our spin-lattice relaxation results are consistent with a coexistence of two types of the rotational motion of $\mathrm{BH}_{4}$ tetrahedra with the activation energies of $0.182 \pm 0.003 \mathrm{eV}$ and 0.251 $\pm 0.004 \mathrm{eV}$. For both types of motions, the jump rates of the reorientations reach the values of the order of $10^{11} \mathrm{~s}^{-1}$ near the upper limit of the temperature range of the orthorhombic phase stability $\left(T_{0} \approx 381 \mathrm{~K}\right)$. In the high-temperature (hexagonal) phase, both the ${ }^{1} \mathrm{H}$ and ${ }^{11} \mathrm{~B}$ spin-lattice relaxation rates are governed by an additional low-frequency fluctuation process (with the characteristic rate of the order of $10^{7} \mathrm{~s}^{-1}$ just above $T_{0}$ ) due to the translational diffusion of $\mathrm{Li}$ ions.
\end{abstract}

\section{Introduction}

The alkali metal borohydrides have received much attention due to their potential as hydrogen storage materials ${ }^{1}$ and energy carriers for fuel cells. ${ }^{2}$ Lithium borohydride $\mathrm{LiBH}_{4}$ containing more than 18 mass percent of hydrogen was recently shown to desorb hydrogen reversibly; ${ }^{3}$ however, its stability with respect to thermal decomposition remains the major drawback for practical use. Elucidation of the complex structure and hydrogen dynamics of $\mathrm{LiBH}_{4}$ may give a key to improving its hydrogenstorage properties by the inclusion of catalytic or destabilizing additives. The structure of $\mathrm{LiBH}_{4}$ was first studied by X-ray diffraction ${ }^{4}$ and more recently by synchrotron X-ray diffraction ${ }^{5-7}$ and neutron diffraction. ${ }^{8}$ The results of these studies show that at low temperatures $\mathrm{LiBH}_{4}$ has an orthorhombic structure and undergoes a first-order phase transition to a hexagonal structure at $T_{0} \approx 381 \mathrm{~K}$. This phase transition has also been detected by Raman scattering ${ }^{9,10}$ and calorimetry. ${ }^{11}$ The transition from the orthorhombic to the hexagonal phase has been found to lead to the 3 orders of magnitude increase in the electrical conductivity, ${ }^{12}$ so that the high-temperature phase of $\mathrm{LiBH}_{4}$ can be considered as a $\mathrm{Li}$ superionic conductor. This conclusion is supported by the ${ }^{7} \mathrm{Li}$ nuclear magnetic resonance (NMR) results for the hexagonal phase of $\mathrm{LiBH}_{4}{ }^{12}$ According to the diffraction data, ${ }^{6}$ the phase transition at $T_{0}$ is also accompanied by a highly anisotropic lattice expansion and a negative volume drop. The experimental data ${ }^{6}$ suggest that the hexagonal structure of the high-temperature phase is stabilized by a nearly isotropic disorder of rigid $\mathrm{BH}_{4}$ tetrahedra. However, little is known about the reorientational motion of the $\mathrm{BH}_{4}$ tetrahedra and the changes in this motion at the phase transition point.

Microscopic details of atomic jump motion in solids can be effectively studied by NMR measurements of the spin relaxation rates. ${ }^{13}$ Previous NMR studies of the $\mathrm{BH}_{4}$ dynamics in $\mathrm{LiBH}_{4}$

\footnotetext{
* Author to whom correspondence should be addressed. E-mail skripov@imp.uran.ru. Fax: +7-343-374-5244.

Ural Division of the Russian Academy of Sciences.

* Swiss-Norwegian Beam Lines at ESRF.
}

include the proton $\left({ }^{1} \mathrm{H}\right)$ spin-lattice relaxation measurements ${ }^{14}$ and the ${ }^{2} \mathrm{D}$ and ${ }^{11} \mathrm{~B}$ spin-lattice relaxation measurements for $\mathrm{LiBH}_{4}\left(\mathrm{D}_{4}\right) .{ }^{15}$ The early proton NMR work ${ }^{14}$ was performed at a single resonance frequency $(19 \mathrm{MHz})$ and in the temperature range $107-375 \mathrm{~K}$, i.e., for the low-temperature phase only. The experimental results for $\mathrm{LiBH}_{4}$ were interpreted ${ }^{14}$ in terms of two inequivalent types of the $\mathrm{BH}_{4}$ tetrahedra; however, this conclusion is not supported by the structural ${ }^{5-8}$ and Raman scattering ${ }^{9,16}$ data. In the present work, we report the results of the proton spin-lattice relaxation measurements in $\mathrm{LiBH}_{4}$ over wide ranges of the resonance frequency $(14-90 \mathrm{MHz})$ and temperature $(92-424 \mathrm{~K})$ complemented by the measurements of the ${ }^{1} \mathrm{H}$ and ${ }^{11} \mathrm{~B}$ NMR spectra and ${ }^{11} \mathrm{~B}$ spin-lattice relaxation rates. The experimental data have been analyzed to determine the parameters of reorientational motion of the $\mathrm{BH}_{4}$ tetrahedra and to assess the changes in this motion associated with the orthorhombic-hexagonal phase transition.

\section{Experimental Methods}

$\mathrm{LiBH}_{4}$ of $99 \%$ purity was purchased from Sigma-Aldrich and used without additional purification. X-ray diffraction analysis at room temperature indicated the presence of a single $\mathrm{LiBH}_{4}$ phase, and no diffraction peaks from impurities were detected. For NMR measurements, the powdered $\mathrm{LiBH}_{4}$ sample was sealed in a glass tube under argon gas. NMR measurements were performed on a modernized Bruker SXP pulse spectrometer at the frequencies $\omega / 2 \pi=14,23.8$, and $90 \mathrm{MHz}\left(\right.$ for ${ }^{1} \mathrm{H}$ ) and 14 and $28 \mathrm{MHz}$ (for ${ }^{11} \mathrm{~B}$ ). A probehead with the sample was placed into an Oxford Instruments CF1200 continuousflow cryostat using nitrogen as a cooling agent. The sample temperature, monitored by a chromel-( $\mathrm{Au}-\mathrm{Fe})$ thermocouple, was stable to $\pm 0.1 \mathrm{~K}$. The nuclear spin-lattice relaxation rates were measured using the saturation-recovery method. In all cases, the recovery of the nuclear magnetization could be satisfactorily described by a single exponential function. NMR spectra were recorded by Fourier transforming the spin echo signals. 


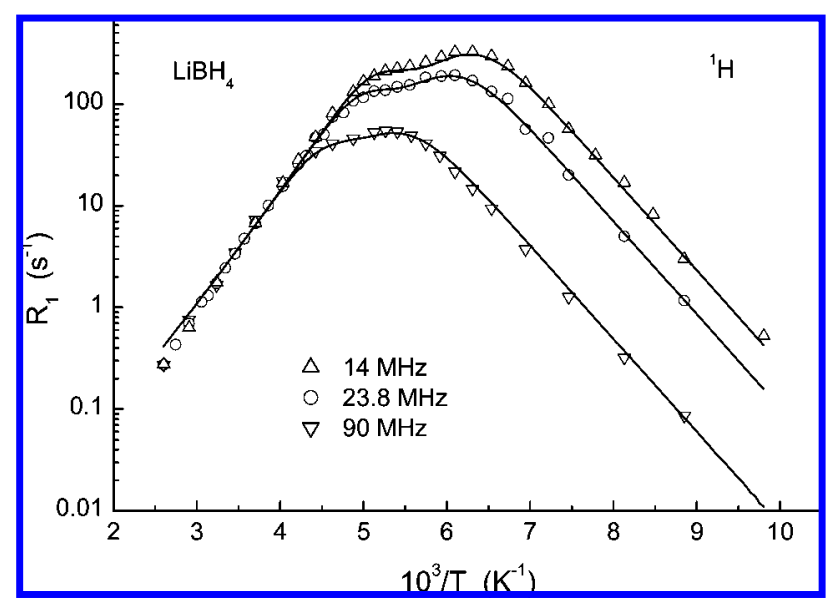

Figure 1. Proton spin-lattice relaxation rates measured at 14, 23.8, and $90 \mathrm{MHz}$ as functions of the inverse temperature for the orthorhombic phase of $\mathrm{LiBH}_{4}$. The solid curves show the simultaneous fits of the model based on eqs $1-3$ to the data.

\section{Results and Discussion}

The temperature dependences of the proton spin-lattice relaxation rates $R_{1}$ measured at three resonance frequencies for the low-temperature phase of $\mathrm{LiBH}_{4}$ are shown in Figure 1. The general features of the observed behavior of $R_{1}$ are typical of the relaxation mechanism due to nuclear dipole-dipole interaction modulated by thermally activated atomic motion. ${ }^{13}$ For this mechanism, the $R_{1}$ maximum is expected to occur at the temperature at which the atomic jump rate $\tau^{-1}$ becomes nearly equal to the resonance frequency $\omega$. In the limit of fast motion $(\omega \tau \ll 1), R_{1}$ should be proportional to $\tau$ being frequency-independent, and in the limit of slow motion $(\omega \tau \gg$ 1 ), $R_{1}$ should be proportional to $\omega^{-2} \tau^{-1}$. If the temperature dependence of $\tau^{-1}$ is governed by the Arrhenius law with the activation energy $E_{\mathrm{a}}$, the plot of $\ln R_{1}$ vs $T^{-1}$ should be linear in the limits of both fast and slow motion with the slopes of $E_{\mathrm{a}} / k_{\mathrm{B}}$ and $-E_{\mathrm{a}} / k_{\mathrm{B}}$, respectively. While the observed behavior of logarithm of $R_{1}$ vs $T^{-1}$ (Figure 1) can be described by linear functions over the $R_{1}$ ranges of at least two decades, the hightemperature slope appears to be steeper than the low-temperature one. This feature of the proton $R_{1}$ data for $\mathrm{LiBH}_{4}$ was also noted by Tsang and Farrar. ${ }^{14}$ In some cases, an asymmetry of the logarithm of the $R_{1}$ vs $T^{-1}$ plot can be accounted for in terms of the model with a continuous distribution of the jump rates $\tau^{-1} \cdot{ }^{17,18}$ However, such a model should also lead to changes in the frequency dependence of $R_{1}$ which becomes weaker than $\omega^{-2}$ in the limit of slow motion. ${ }^{17-19}$ Since in our case the frequency dependence of $R_{1}$ at the low-temperature slope of the $R_{1}$ peak is well described by $\omega^{-2}$, we can reject the model with a continuous distribution of the jump rates. Furthermore, the data presented in Figure 1 exhibit the inflection point near $180 \mathrm{~K}$; this suggests that the observed temperature dependence of $R_{1}$ can be described as a superposition of two peaks with different slopes. It has been assumed ${ }^{14}$ that in $\mathrm{LiBH}_{4}$ there are two inequivalent types of $\mathrm{BH}_{4}$ tetrahedra rotating at different frequencies. However, the existence of two types of $\mathrm{BH}_{4}$ tetrahedra is not supported by the diffraction results for $\mathrm{LiBH}_{4} .^{5-8}$ Our interpretation of the $R_{1}$ data for the lowtemperature phase of $\mathrm{LiBH}_{4}$ is based on the assumption that each $\mathrm{BH}_{4}$ tetrahedron participates in two types of rotational motion (most probably, 2-fold and 3-fold jump rotations) having different rates. We will use the subscript $i(i=1,2)$ to denote the two types of motion assuming that $i=1$ corresponds to the faster motion (i.e., the one giving rise to the $R_{1}$ peak at lower
T). To estimate the relative strength of the ${ }^{1} \mathrm{H}-{ }^{11} \mathrm{~B},{ }^{1} \mathrm{H}-{ }^{1} \mathrm{H}$, and ${ }^{1} \mathrm{H}-{ }^{7} \mathrm{Li}$ dipole-dipole interactions, we have calculated the corresponding contributions to the "rigid lattice" second moment of the ${ }^{1} \mathrm{H}$ NMR line on the basis of the structural data ${ }^{6}$ for the low-temperature phase taking into account internuclear distances up to $4 \AA$. The resulting rigid lattice contributions are $M \mathrm{AB}_{\mathrm{B}}=$ $1.36 \times 10^{10} \mathrm{~s}^{-2}, M_{\mathrm{HH}}^{R}=1.59 \times 10^{10} \mathrm{~s}^{-2}$, and $M_{\mathrm{HLi}}^{R}=2.01 \times$ $10^{9} \mathrm{~s}^{-2}$. Thus, the $\mathrm{H}-\mathrm{B}$ and $\mathrm{H}-\mathrm{H}$ interactions are of nearly equal strength, while the $\mathrm{H}-\mathrm{Li}$ interactions are an order of magnitude weaker. For the motion characterized by the jump rate $\tau_{i}^{-1}$, the proton spin-lattice relaxation rate is dominated by the sum of contributions due to $\mathrm{H}-\mathrm{B}$ and $\mathrm{H}-\mathrm{H}$ dipole-dipole interactions,

$$
\begin{aligned}
& R_{1 i}=\frac{\Delta M_{\mathrm{HB} i} \tau_{i}}{2}\left[\frac{1}{1+\left(\omega_{\mathrm{H}}-\omega_{\mathrm{B}}\right)^{2} \tau_{i}^{2}}+\frac{3}{1+\omega_{\mathrm{H}}^{2} \tau_{i}^{2}}+\right. \\
& \left.\frac{6}{1+\left(\omega_{\mathrm{H}}+\omega_{\mathrm{B}}\right)^{2} \tau_{i}^{2}}\right]+\frac{4 \Delta M_{\mathrm{HH} i} \tau_{i}}{3}\left[\frac{1}{4+\omega_{\mathrm{H}}^{2} \tau_{i}^{2}}+\frac{1}{1+\omega_{\mathrm{H}}^{2} \tau_{i}^{2}}\right]
\end{aligned}
$$

where $\omega_{\mathrm{H}}$ and $\omega_{\mathrm{B}}$ are the resonance frequencies of ${ }^{1} \mathrm{H}$ and ${ }^{11} \mathrm{~B}$, respectively, and $\Delta M_{\mathrm{HB} i}$ and $\Delta M_{\mathrm{HH} i}$ are the parts of the dipolar second moment due to $\mathrm{H}-\mathrm{B}$ and $\mathrm{H}-\mathrm{H}$ interactions that are caused to fluctuate by the $i$ th type of motion. We assume that for each type of motion the temperature dependence of $\tau_{i}^{-1}$ is governed by the Arrhenius law

$$
\tau_{i}^{-1}=\tau_{0 i}^{-1} \exp \left(-E_{\mathrm{a} i} / k_{\mathrm{B}} T\right)
$$

and

$$
R_{1}=R_{11}+R_{12}
$$

The parameters of the model are $\Delta M_{\mathrm{HB} i}, \Delta M_{\mathrm{HH} i}, \tau_{0 i}$, and $E_{\mathrm{a} i}$. These parameters are varied to find the best fit to the $R_{1}(T)$ data at the three resonance frequencies simultaneously. Since the $\mathrm{H}-\mathrm{B}$ and $\mathrm{H}-\mathrm{H}$ terms in eq 1 show nearly the same temperature and frequency dependences, it is practically impossible to determine the amplitude parameters $\Delta M_{\mathrm{HB} i}$ and $\Delta M_{\mathrm{HH} i}$ independently from the fits. Therefore, we have to assume that the ratio $\Delta M_{\mathrm{HB} i} / \Delta M_{\mathrm{HH} i}$ is nearly the same as for the corresponding contributions to the rigid lattice dipolar second moment.

The results of the simultaneous fit based on eqs $1-3$ are shown by solid curves in Figure 1. As can be seen from this figure, the model gives a good description of the experimental data at three resonance frequencies. The values of the amplitude parameters resulting from the fits are $\Delta M_{\mathrm{HB} 1}=8.2 \times 10^{9} \mathrm{~s}^{-2}$, $\Delta M_{\mathrm{HH} 1}=9.6 \times 10^{9} \mathrm{~s}^{-2}, \Delta M_{\mathrm{HB} 2}=4.0 \times 10^{9} \mathrm{~s}^{-2}$, and $\Delta M_{\mathrm{HH} 2}$ $=4.6 \times 10^{9} \mathrm{~s}^{-2}$, and the corresponding motional parameters are $\tau_{01}=(1.9 \pm 0.1) \times 10^{-14} \mathrm{~s}, E_{\mathrm{a} 1}=0.182 \pm 0.003 \mathrm{eV}, \tau_{02}$ $=(3.1 \pm 0.2) \times 10^{-15} \mathrm{~s}$, and $E_{\mathrm{a} 2}=0.251 \pm 0.004 \mathrm{eV}$. The activation energies reported in ref 14 are, in our notations, $E_{\mathrm{a} 1}$ $=0.17 \pm 0.01$ and $E_{\mathrm{a} 2}=0.21 \pm 0.01 \mathrm{eV}$. While for $E_{\mathrm{a} 1}$ the agreement is satisfactory, our $E_{\mathrm{a} 2}$ value appears to exceed that of ref 14 . It should be noted that, in contrast to ref 14 , our analysis is based on the data at three different resonance frequencies and includes the broader range of $R_{1}$ values than that of ref 14. Furthermore, as will be shown below, the values of $E_{\mathrm{a} 1}$ and $E_{\mathrm{a} 2}$ derived from our analysis of the proton $R_{1}$ are very close to those that describe the behavior of the ${ }^{11} \mathrm{~B}$ spin-lattice relaxation rate for the low-temperature phase of $\mathrm{LiBH}_{4}$. For the case of isolated $\mathrm{BH}_{4}$ tetrahedra, it is possible to estimate the parts of the dipolar second moment due to $\mathrm{H}-\mathrm{B}$ and $\mathrm{H}-\mathrm{H}$ interactions that are caused to fluctuate by the 2-fold and 3 -fold reorientations. Using the expressions derived in ref 20 , for the 2 -fold reorientations, we obtain $\Delta M_{\mathrm{HB}}^{\mathrm{f}}=8.4 \times 10^{9}$ 


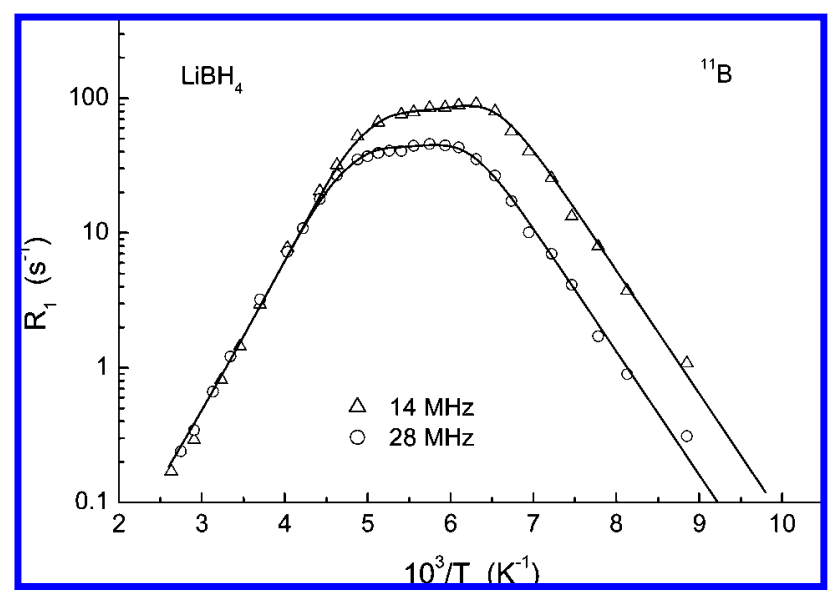

Figure 2. ${ }^{11} \mathrm{~B}$ spin-lattice relaxation rates measured at 14 and $28 \mathrm{MHz}$ as functions of the inverse temperature for the orthorhombic phase of $\mathrm{LiBH}_{4}$. The solid curves show the simultaneous fits of the model based on eqs 4,2 , and 3 to the data.

$\mathrm{s}^{-2}$ and $\Delta M_{\mathrm{HH}}^{\mathrm{f}}=5.6 \times 10^{9} \mathrm{~s}^{-2}$. The analogous approach for the 3 -fold reorientations yields $\Delta M_{\mathrm{HB}}^{3 \mathrm{f}}=8.3 \times 10^{9} \mathrm{~s}^{-2}$ and $\Delta M_{\mathrm{HH}}^{3 \mathrm{f}}=8.5 \times 10^{9} \mathrm{~s}^{-2}$. These values are of the same order of magnitude as those obtained from the proton $R_{1}$ fits. However, such estimates can hardly be used to identify the type of reorientations corresponding to each of the $R_{1}$ peaks. More direct methods, such as quasielastic neutron scattering, are required to solve this problem.

The temperature dependences of the ${ }^{11} \mathrm{~B}$ spin-lattice relaxation rates measured at two resonance frequencies for the lowtemperature phase of $\mathrm{LiBH}_{4}$ are shown in Figure 2. Comparison of Figures 1 and 2 indicates that general features of the behavior of the ${ }^{11} \mathrm{~B}$ relaxation rates are similar to those of the proton relaxation rates. In particular, the model with two coexisting types of rotational motion of $\mathrm{BH}_{4}$ tetrahedra seems to be applicable to the ${ }^{11} \mathrm{~B}$ data. Since, in contrast to protons, ${ }^{11} \mathrm{~B}$ nuclei have nonzero electric quadrupole moments, one may expect that the interaction between the nuclear quadrupole moments and fluctuating local electric field gradients ${ }^{13}$ contributes to the ${ }^{11} \mathrm{~B}$ relaxation rate (in addition to the dipole-dipole $\mathrm{B}-\mathrm{H}$ interaction). Thus, for the motion characterized by the jump rate $\tau_{i}^{-1}$, the ${ }^{11} \mathrm{~B}$ spin-lattice relaxation rate can be expressed as a sum of the quadrupolar and dipolar terms

$$
\begin{aligned}
R_{1 i}=\Delta M_{\mathrm{Q} i} \tau_{i}\left[\frac{1}{1+\omega_{\mathrm{B}}^{2} \tau_{i}^{2}}+\frac{4}{1+4 \omega_{\mathrm{B}}^{2} \tau_{i}^{2}}\right]+\frac{\Delta M_{\mathrm{BH} i} \tau_{i}}{2} \times \\
\\
{\left[\frac{1}{1+\left(\omega_{\mathrm{B}}-\omega_{\mathrm{H}}\right)^{2} \tau_{i}^{2}}+\frac{3}{1+\omega_{\mathrm{B}}^{2} \tau_{i}^{2}}+\frac{6}{1+\left(\omega_{\mathrm{B}}+\omega_{\mathrm{H}}\right)^{2} \tau_{i}^{2}}\right] }
\end{aligned}
$$

where the amplitude parameter $\Delta M_{\mathrm{Q} i}$ is proportional to the square of the electric quadrupole moment of ${ }^{11} \mathrm{~B}$ and the square of the fluctuating part of the electric field gradient at ${ }^{11} \mathrm{~B}$ sites due to $i$ th type of motion. As in the case of eq 1, the two terms in eq 4 show nearly the same temperature and frequency dependences; therefore, it is practically impossible to determine the amplitude parameters $\Delta M_{\mathrm{Q} i}$ and $\Delta M_{\mathrm{BH} i}$ independently from the fits. Comparison of the ${ }^{11} \mathrm{~B}$ spin-lattice relaxation data for $\mathrm{LiBH}_{4}$ and $\mathrm{LiBD}_{4}{ }^{15}$ shows that the quadrupolar contribution to the amplitude of the $R_{1}$ peak in $\mathrm{LiBH}_{4}$ does not exceed $10 \%$. Indeed, the quadrupolar contribution to the ${ }^{11} \mathrm{~B}$ spin-lattice relaxation rate should be the same for $\mathrm{LiBH}_{4}$ and $\mathrm{LiBD}_{4}$ (since only charge fluctuations are important for the quadrupolar mechanism of relaxation), whereas the maximum $R_{1}$ value for ${ }^{11} \mathrm{~B}$ in $\mathrm{LiBH}_{4}$ exceeds that in $\mathrm{LiBD}_{4}{ }^{15}$ by nearly a factor of 10 .

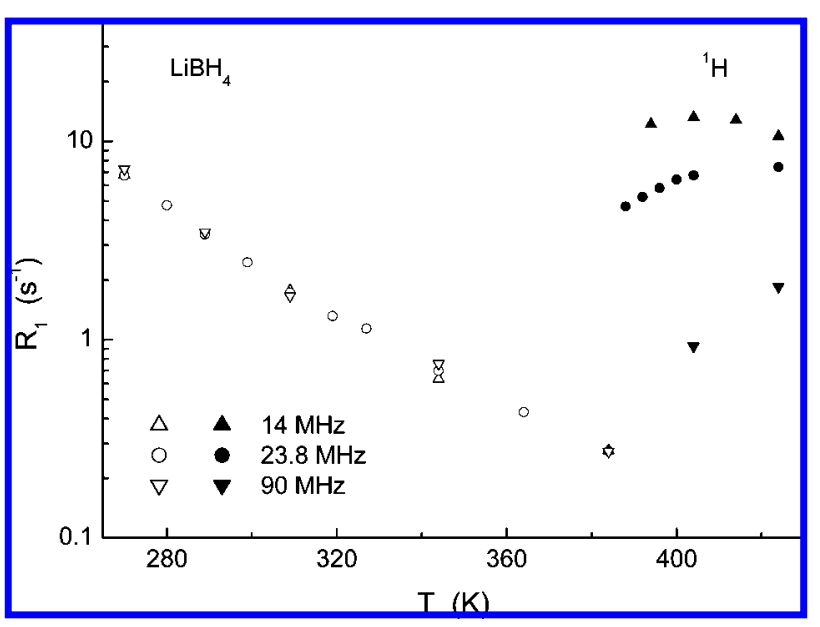

Figure 3. Behavior of the proton spin-lattice relaxation rates measured at $14,23.8$, and $90 \mathrm{MHz}$ below and above the temperature of the structural phase transition in $\mathrm{LiBH}_{4}$. Open symbols correspond to the orthorhombic phase, and solid symbols correspond to the hexagonal phase.

On the basis of these considerations, we have fixed the parameters $\Delta M_{\mathrm{Q} i}$ to the values giving about $10 \%$ of the corresponding $R_{1 i}$ peak amplitudes. Such a procedure does not affect the values of $E_{\mathrm{a} i}$ resulting from the fits. Again, we look for a set of parameters giving the best fit to the $R_{1}(T)$ data at two resonance frequencies simultaneously. The results of the simultaneous fits based on eqs 4, 2, and 3 are shown by solid curves in Figure 2. The amplitude parameters resulting from the fit are $\Delta M_{Q 1}=1.0 \times 10^{9} \mathrm{~s}^{-2}, \Delta M_{\mathrm{BH} 1}=5.8 \times 10^{9} \mathrm{~s}^{-2}$, $\Delta M_{\mathrm{Q} 2}=5.1 \times 10^{8} \mathrm{~s}^{-2}$, and $\Delta M_{\mathrm{BH} 2}=3.0 \times 10^{9} \mathrm{~s}^{-2}$, and the motional parameters are $\tau_{01}=(1.5 \pm 0.1) \times 10^{-14} \mathrm{~s}, E_{\mathrm{a} 1}=$ $0.182 \pm 0.003 \mathrm{eV}, \tau_{02}=(1.6 \pm 0.1) \times 10^{-15} \mathrm{~s}$, and $E_{\mathrm{a} 2}=$ $0.256 \pm 0.003 \mathrm{eV}$. Note that these values of $E_{\mathrm{a} 1}$ and $E_{\mathrm{a} 2}$ are very close to the corresponding values derived from our analysis of the proton relaxation rates. For comparison, the value of the single activation energy obtained from the ${ }^{11} \mathrm{~B}$ spin-lattice relaxation data in ref 15 is $0.21 \mathrm{eV}$. However, this estimate is based on the data in the limited temperature range of 220-330 $\mathrm{K}$. Our analysis is based on the data at two different resonance frequencies and includes broader ranges of the temperature and $R_{1}$.

Note that both the ${ }^{1} \mathrm{H}$ and ${ }^{11} \mathrm{~B}$ spin-lattice relaxation rates for the orthorhombic phase of $\mathrm{LiBH}_{4}$ behave according to the expectations for two types of independent thermally activated jump motion. We have not found any correlation between $R_{1}(T)$ and the anomalous lattice expansion observed by diffraction, ${ }^{6}$ neither above $300 \mathrm{~K}$ where the cell parameter $b$ shows a strong negative thermal expansion nor below $150 \mathrm{~K}$ where the cell parameter $a$ increases on cooling. Therefore, the anomalies in the thermal expansion are not related to the rotational motion of the $\mathrm{BH}_{4}$ tetrahedra.

The behavior of the ${ }^{1} \mathrm{H}$ and ${ }^{11} \mathrm{~B}$ spin-lattice relaxation rates below and above the temperature of the structural phase transition in $\mathrm{LiBH}_{4}$ is shown in Figures 3 and 4. As can be seen from these figures, the transition from the low-temperature orthorhombic phase to the high-temperature hexagonal phase leads to a sharp increase in both ${ }^{1} \mathrm{H}$ and ${ }^{11} \mathrm{~B}$ relaxation rates. According to our data, the transition occurs in the range $381-384 \mathrm{~K}$. The ${ }^{11} \mathrm{~B}$ relaxation rate data reported in ref 15 exhibit a much broader transition range (of the order of $20 \mathrm{~K}$ ). This suggests the existence of a large temperature gradient through the sample volume in the earlier experiments. ${ }^{15}$ The most interesting feature of our relaxation rate data for the high- 


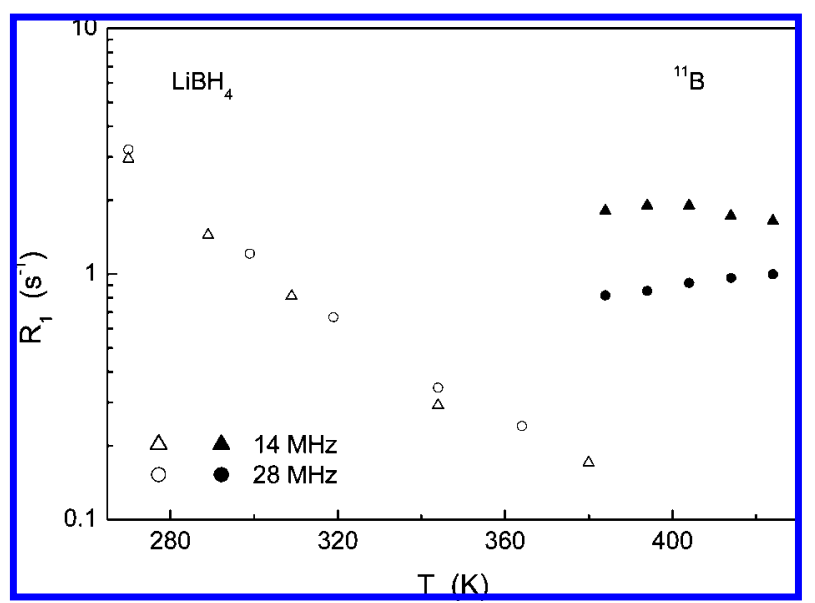

Figure 4. Behavior of the ${ }^{11} \mathrm{~B}$ spin-lattice relaxation rates measured at 14 and $28 \mathrm{MHz}$ below and above the temperature of the structural phase transition in $\mathrm{LiBH}_{4}$. Open symbols correspond to the orthorhombic phase, and solid symbols correspond to the hexagonal phase.

temperature phase of $\mathrm{LiBH}_{4}$ is the onset of pronounced frequency dependence of $R_{1}$ for both ${ }^{1} \mathrm{H}$ and ${ }^{11} \mathrm{~B}$ (Figures 3 and 4). Note that for the low-temperature phase in the range $230-380 \mathrm{~K}$ the spin-lattice relaxation rates are frequencyindependent, since the jump rates $\tau_{i}{ }^{-1}$ in this range become much higher than the resonance frequencies $\left(\omega \tau_{i} \ll 1\right)$. The reappearance of the frequency dependence of $R_{1}$ for the high-temperature phase means that above $T_{0}$ the spin-lattice relaxation is governed by much slower fluctuations than below $T_{0}$. In fact, from the Arrhenius fits to the data for the low-temperature phase, we obtain $\tau_{1}^{-1}(380 \mathrm{~K})=2.1 \times 10^{11} \mathrm{~s}^{-1}$ and $\tau_{2}^{-1}(380 \mathrm{~K})=1.5$ $\times 10^{11} \mathrm{~s}^{-1}$. On the other hand, the frequency and temperature dependences of $R_{1}$ in the range 384-424 K (Figures 3 and 4) suggest that just above the transition point the jump rate $\tau_{\mathrm{d}}{ }^{-1}$ responsible for the spin-lattice relaxation is somewhat lower than the lowest of our $\omega$ values, i.e., $\tau_{\mathrm{d}}{ }^{-1}(384 \mathrm{~K})<8.8 \times 10^{7}$ $\mathrm{s}^{-1}$. Since it is unlikely that the jump rates of the $\mathrm{BH}_{4}$ rotations drop by more than 3 orders of magnitude with increasing temperature, we may expect the onset of some additional lowfrequency fluctuation processes above $T_{0}$. Taking into account the ${ }^{7} \mathrm{Li} R_{1}$ data, ${ }^{12}$ it seems reasonable to assume that the translational diffusion of Li ions plays the role of such a process in the high-temperature phase. Although the $\mathrm{H}-\mathrm{Li}$ dipolar coupling is considerably weaker than the $\mathrm{H}-\mathrm{H}(\mathrm{B})$ dipolar coupling, the former can still give the dominant contribution to the proton $R_{1}$, if the rotational jump rates $\tau_{1}{ }^{-1}$ and $\tau_{2}{ }^{-1}$ are much higher than the resonance frequency $\omega$, while the Li jump rate $\tau_{\mathrm{d}}^{-1}$ is of the order of $\omega$. To estimate the maximum contributions to the ${ }^{1} \mathrm{H}$ and ${ }^{11} \mathrm{~B}$ spin-lattice relaxation rates due to $\mathrm{Li}$ diffusion, on the basis of the structural data ${ }^{6}$ for the hightemperature phase, we have calculated the $\mathrm{H}-\mathrm{Li}$ and $\mathrm{B}-\mathrm{Li}$ contributions to the rigid lattice dipolar second moments of the ${ }^{1} \mathrm{H}$ and ${ }^{11} \mathrm{~B}$ NMR lines, respectively. The corresponding values are $M_{\mathrm{ALi}}^{R}=1.32 \times 10^{9} \mathrm{~s}^{-2}$ and $M_{\mathrm{BLi}}^{R}=1.18 \times 10^{8} \mathrm{~s}^{-2}$. If these contributions are fully modulated by Li diffusion, the expected maximum spin-lattice relaxation rates at $\omega / 2 \pi=14 \mathrm{MHz}$ are $32.2 \mathrm{~s}^{-1}$ for ${ }^{1} \mathrm{H}$ and $2.34 \mathrm{~s}^{-1}$ for ${ }^{11} \mathrm{~B}$. Our experimental $R_{1}$ values for both ${ }^{1} \mathrm{H}$ and ${ }^{11} \mathrm{~B}$ in the high-temperature phase (see Figures 3 and 4) are somewhat lower than these calculated maximum values. This allows us to conclude that the $\mathrm{H}-\mathrm{Li}$ and $\mathrm{B}-\mathrm{Li}$ dipolar coupling is strong enough to give rise to the observed relaxation rates in the hexagonal phase. Using the Arrhenius fit to the ${ }^{7} \mathrm{Li} R_{1}$ data from ref 12 , we find that the $\mathrm{Li}$ jump rate just

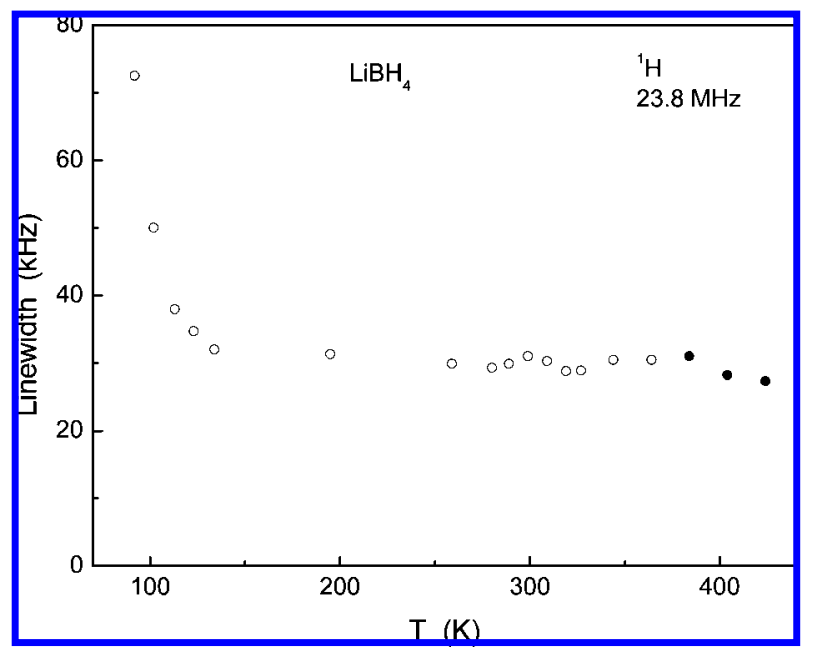

Figure 5. Temperature dependence of the width (full width at halfmaximum) of the proton NMR spectrum measured at $23.8 \mathrm{MHz}$ for $\mathrm{LiBH}_{4}$. Open symbols correspond to the orthorhombic phase, and solid symbols correspond to the hexagonal phase.

above the transition point is $\tau_{\mathrm{d}}{ }^{-1}(384 \mathrm{~K}) \approx 4.2 \times 10^{7} \mathrm{~s}^{-1}$. This value is consistent with the above estimate $\left(\tau_{\mathrm{d}}^{-1}(384 \mathrm{~K})<8.8\right.$ $\times 10^{7} \mathrm{~s}^{-1}$ ) based on our ${ }^{1} \mathrm{H}$ and ${ }^{11} \mathrm{~B}$ relaxation data. Thus, the frequency range of the Li jump motion also agrees with our results for the high-temperature phase of $\mathrm{LiBH}_{4}$. However, we cannot make any conclusions about the rotational motion of the $\mathrm{BH}_{4}$ tetrahedra in the high-temperature phase on the basis of our ${ }^{1} \mathrm{H}$ and ${ }^{11} \mathrm{~B}$ spin-lattice relaxation data.

Additional information on the motion of the $\mathrm{BH}_{4}$ tetrahedra can, in principle, be obtained from the behavior of the ${ }^{1} \mathrm{H}$ and ${ }^{11} \mathrm{~B}$ NMR spectra. Figure 5 shows the temperature dependence of the ${ }^{1} \mathrm{H}$ line width (full width at half-maximum) in the range $92-424 \mathrm{~K}$. The sharp narrowing of the line is observed near $100 \mathrm{~K}$. Usually the narrowing becomes pronounced above the temperature at which the jump rate exceeds the rigid-lattice (lowtemperature) line width. ${ }^{13}$ Since the dipolar rigid-lattice line width is much smaller than the resonance frequency, the line narrowing should occur at considerably lower temperatures than the $R_{1}$ maximum, in agreement with our experimental results (Figures 1 and 5). As the temperature increases, the proton line width stops to decrease being close to $30 \mathrm{kHz}$ over the temperature range 130-424 K (Figure 5). This rather high plateau value indicates that the motion responsible for the line narrowing is indeed localized since such a motion leads to only partial averaging of the dipole-dipole interactions. The transition from the orthorhombic to the hexagonal phase is not accompanied by a considerable change in the proton line width. This suggests that the $\mathrm{BH}_{4}$ tetrahedra do not participate in the long-range diffusion, at least just above $T_{0}$, so that $\mathrm{Li}$ ions are the only diffusing species, in agreement with the conclusions of ref 12 . On the other hand, the $\mathrm{H}-\mathrm{Li}$ dipolar interaction is not strong enough to cause a considerable narrowing of the ${ }^{1} \mathrm{H}$ NMR line due to Li diffusion.

The evolution of the measured ${ }^{11} \mathrm{~B}$ NMR spectrum with temperature is shown in Figure 6. As can be seen from this figure, in the low-temperature phase the spectrum consists of two components: a narrow central line and broader "wings" which can be attributed to unresolved quadrupole satellites. It should be noted that these wings are observed up to the transition point. This means that the environment of the ${ }^{11} \mathrm{~B}$ nuclei is noncubic, and the quadrupole interaction of ${ }^{11} \mathrm{~B}$ is not averaged out by the fast rotational motion in the low-temperature phase of $\mathrm{LiBH}_{4}$. Such a situation is possible if each elementary 


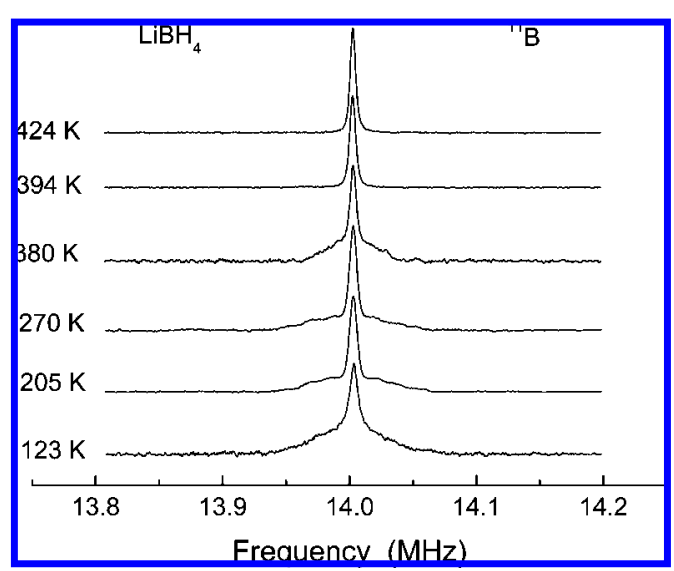

Figure 6. Evolution of the ${ }^{11} \mathrm{~B}$ NMR spectrum measured at $14 \mathrm{MHz}$ with the temperature.

rotational jump of a $\mathrm{BH}_{4}$ tetrahedron does not change the local electric field gradient at a boron site. This is what is expected to occur in the cases of the 3-fold and 2-fold reorientations of rigid $\mathrm{BH}_{4}$ tetrahedra. The absence of the motional modulation of the ${ }^{11} \mathrm{~B}$ quadrupole interaction in the low-temperature phase of $\mathrm{LiBH}_{4}$ is also consistent with the small upper limit of the quadrupolar contribution to the ${ }^{11} \mathrm{~B}$ spin-lattice relaxation rate (see the discussion above). In the high-temperature phase of $\mathrm{LiBH}_{4}$, the wings of the ${ }^{11} \mathrm{~B}$ NMR spectrum completely disappear (Figure 6). This suggests a cubic environment of ${ }^{11} \mathrm{~B}$ nuclei. In fact, according to the structural data, ${ }^{6,8}$ the $\mathrm{BH}_{4}$ groups in the high-temperature phase are very close to the ideal tetrahedra. Another possible explanation of the disappearance of the wings in the hexagonal phase is related to deviations from the ideal angles of rotation. If a rotational jump does not lead to exactly the same configuration of a $\mathrm{BH}_{4}$ group, the quadrupole interaction of ${ }^{11} \mathrm{~B}$ nuclei may be dynamically averaged to zero. Such a behavior is expected to result in the increase in the mean-square atomic displacement parameters (ADPs) seen by diffraction measurements. In fact, the results of both synchrotron and neutron diffraction experiments ${ }^{6,8}$ have revealed an abrupt increase in the ADPs of the $\mathrm{BH}_{4}$ groups upon the transition from the orthorhombic to the hexagonal phase.

\section{Conclusions}

The analysis of the temperature and frequency dependences of our ${ }^{1} \mathrm{H}$ and ${ }^{11} \mathrm{~B}$ spin-lattice relaxation data has shown that in the low-temperature (orthorhombic) phase of $\mathrm{LiBH}_{4}$ there are two coexisting types of rotational motion of the $\mathrm{BH}_{4}$ tetrahedra. These types of motion (most probably, the 2-fold and 3-fold reorientations) are characterized by the activation energies of $0.182 \pm 0.003 \mathrm{eV}$ and $0.251 \pm 0.004 \mathrm{eV}$. For both types of motions, the jump rates of the reorientations reach the values of the order of $10^{11} \mathrm{~s}^{-1}$ near the upper limit of the temperature range of the orthorhombic phase stability $\left(T_{0} \approx\right.$ $381 \mathrm{~K})$. It has been found that the structural transition from the orthorhombic to the high-temperature hexagonal phase of $\mathrm{LiBH}_{4}$ leads to a sharp increase in both ${ }^{1} \mathrm{H}$ and ${ }^{11} \mathrm{~B}$ spin-lattice relaxation rates and to a reappearance of their frequency dependence. These results indicate that in the high-temperature phase the measured ${ }^{1} \mathrm{H}$ and ${ }^{11} \mathrm{~B}$ spin-lattice relaxation rates are governed by an additional low-frequency fluctuation process (with the characteristic rate of the order of $10^{7} \mathrm{~s}^{-1}$ just above $T_{0}$ ) due to translational diffusion of $\mathrm{Li}$ ions, while the contributions due to the rotational motion of $\mathrm{BH}_{4}$ tetrahedra are small in this temperature range.

Acknowledgment. The authors are grateful to the anonymous reviewers for useful suggestions. This work was partially supported by the Priority Program "Basic energy problems" of the Russian Academy of Sciences.

\section{References and Notes}

(1) Orimo, S.; Nakamori, Y.; Eliseo, J. R.; Züttel, A.; Jensen, C. M. Chem. Rev. 2007, 107, 4111.

(2) Li, Z. P.; Liu, B. H.; Morigasaki, N.; Suda, S. J. Allovs Compd. 2003, 354, 243.

(3) Mauron, P.; Buchter, F.; Friedrichs, O.; Remhof, A.; Bielmann, M.; Zwicky, C. N.; Züttel, A. J. Phvs. Chem. B 2008, 112, 906.

(4) Harris, P. M.; Meibohm, E. M. J. Am. Chem. Soc. 1947, 69, 1231.

(5) Soulié, J.-P.; Renaudin, G.; Črný, R.; Yvon, K. J. Alloys Compd. 2002, 346, 200.

(6) Filinchuk, Y.; Chernyshov, D.; Černý, R. J.Phys. Chem.C 2008, $112,10579$.

(7) Dmitriev, V.; Filinchuk, Y.; Chernyshov, D.; Talyzin, A. V.; Dzwilewski, A.; Andersson, O.; Sundqvist, B.; Kurnosov, A. Phvs. Rev. B 2008, 77, 174112 .

(8) Hartman, M. R.; Rush, J. J.; Udovic, T. J.; Bowman, R. C.; Hwang, S.-J. J. Solid State Chem. 2007, 180, 1298. 206.

(9) Gomes, S.; Hagemann, H.; Yvon, K. J. Allovs Compd. 2002, 346,

(10) Nakamori, Y.; Orimo, S. J. Allovs Compd. 2004, 370, 271

(11) Gavrichev, K. S. Inorg. Mater. 2003, 39, S89.

(12) Matsuo, M.; Nakamori, Y.; Orimo, S.; Maekawa, H.; Takamura, H. Appl. Phys. Lett. 2007, 91, 224103.

(13) Abragam, A. The Principles of Nuclear Magnetism; Clarendon Press: Oxford, 1961

(14) Tsang, T.; Farrar, T. C. J. Chem. Phvs. 1969, 50, 3498.

(15) Tarasov, V. P.; Bakum, S. I.; Privalov, V. I.; Shamov, A. A. Russ. J. Inorg. Chem. 1990, 35, 1034.

(16) Hagemann, H.; Gomes, S.; Renaudin, G.; Yvon, K. J. Allovs Compd. 2004, 363, 126.

(17) Shinar, J.; Davidov, D.; Shaltiel, D. Phvs. Rev. B 1984, 30, 6331. (18) Markert, J. T.; Cotts, E. J.; Cotts, R. M. Phvs. Rev. B 1988, 37 , 6446.

(19) Skripov, A. V.; Rychkova, S. V.; Belyaev, M. Yu.; Stepanov, A. P. Solid State Commun. 1989, 71, 1119.

(20) Dereppe, J. M. J. Chem. Phvs. 1973, 58, 1254.

JP806705Q 